MODELING, IDENTIFICATION AND CONTROL, 1995, VOL. 16, NO. 1, 35-49

doi:10.4173/mic.1995.1.2

\title{
Robust Control and Analysis of a Wind-Diesel Hybrid Power Plant
}

\author{
K. UHLEN*†, B. A. FOSS $†$ and O. B. GJøSÆTER $\dagger$
}

Keywords: Autonomous power systems, wind-diesel systems, robust control, multivariable frequency analysis, decoupling, LQC/oop transfer recovery.

The aim of this paper is twofold. First to present multivariable frequency domain techniques as a tool for controller design and dynamic analysis of an autonomous wind-diesel power system. Secondly to study how robust model based controllers can be designed for such systems.

Dynamic system analyses using multivariable frequency domain techniques are verified against detailed nonlinear simulation studies.

The results are encouraging in the sense that the main conclusions in terms of robust stability and performance agree very well with the simulation results. It is also shown that improved performance of the system can be achieved using simple model based controllers.

\section{Introduction}

An important application for renewable energy sources, like wind energy converters (WECs), is in conjunction with autonomous power systems. Today, diesel generator sets are the dominating source of electric power in remote regions. Stand-alone wind-diesel hybrid systems represent, economically, an interesting alternative for communities, typically islands, with good wind conditions.

However, there is a reluctance among potential users to install wind-diesel systems, or other hybrid solutions. A main reason for this, is uncertainty with respect to reliability and operational costs of these technically more complex systems. Another reason may be uncertainty with respect to power quality and robustness of such systems, which rely heavily on the rapidly fluctuating power from the wind.

The aim of this paper is to investigate robustness and power quality performance of a simple wind-diesel system. The study is based on a real system consisting of a $55 \mathrm{~kW}$ standard stall-regulated wind turbine generator, operating in parallel with a $50 \mathrm{~kW}$ diesel generator set, and serving a common resistive consumer load. The main objective of the paper is twofold: First, to illustrate how multivariable frequency domain techniques can be applied to the analysis of power systems, and secondly to analyse how different control structures affect the performance and stability of the wind-diesel system.

\footnotetext{
Received 13 September 1994.

* The Norwegian Electric Power Research Institute (EFI) N-7034 Trondheim, Email: kju@efi.sintef.no.

$\dagger$ The University of Trondheim, The Norwegian Institute of Technology (NTH), Department of Engineering Cybernetics, N-7034 Trondheim, Norway.

(C) IEEE. Presented at the IEEE/PES 1994 Winter Meeting, New York, New York, January 30-February 3, 1994.

Reprinted with permission from the IEEE.
} 
The choice of controllers is motivated by the fact that the system, from a control point of view, essentially is a $2 \times 2$ system. We manipulate the field voltage of the synchronous generator and fuel flow input to the diesel engine to control the voltage and frequency of the power grid. In addition, the system is nonlinear. Three different control structures are chosen for investigation, of which two are model based. The first is a robust approach to feedback linearization and decoupling, where the controller is based on a reduced order (possibly nonlinear) model. The second is a robust approach to a LQG-optimal controller, implemented using the loop transfer recovery (LTR) design methodology (Athans (1986)). The third controller, which is included for comparison purposes, consisting of single loop PI-controllers.

The analysis of the plant model and the different closed loop systems are performed partly by means of linearized system analysis, such as singular value frequency responses, and partly by system simulations, using a newly developed nonlinear simulation model (Lundsager et al. (1991)). The simulation model has been validated against data from the real system. An important motivation for this study has been to investigate how linear robust stability and performance analyses conform with results obtained by more accurate simulation studies.

The paper is organized as follows. First, there is a short introduction to wind-diesel hybrid power systems, and a brief description of the dynamic model of the system chosen for study. The next section deals with control objectives, motivation and choice of control structures. The main section describes the system analyses, and cover plant analysis, controller design and closed loop system analysis. Nonlinear system simulation results are presented for comparison purposes before the concluding remarks.

\section{Modeling and description of the basic wind-diesel system}

There are numerous ways of designing hybrid autonomous power systems combining renewable energy sources and firm backup power from e.g. diesel generator sets. An overview of recent development of wind-diesel systems, in particular, can be found in Infield et al. (1992). A basic wind-diesel system, illustrated in Fig. 1 is chosen for this study. The system consists of a three-bladed $16 \mathrm{~m}$ diameter stall-regulated wind turbine with an induction generator rated at $55 \mathrm{~kW}$. The diesel generator set comprises a $50 \mathrm{~kW}$ turbocharged diesel engine and a $65 \mathrm{kVA}$ brushless synchronous generator. The two generators are connected to a common ac bus-bar, serving a distributed consumer load. The operating philosophy is to let the wind turbine produce as much as possible of the power demand, while the diesel generator covers the deficit, $\boldsymbol{P}_{\text {sg }}=\boldsymbol{P}_{\text {load }}-\boldsymbol{P}_{\text {ag. }}$. The actual system is part of a prototype wind-diesel system described

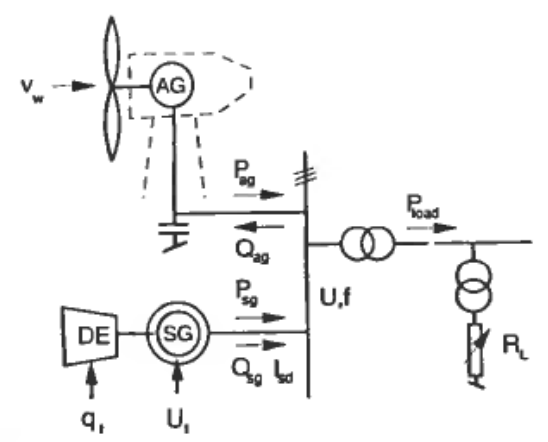

Figure 1. Basic wind-diesel system. 
in Uhlen, Skarstein, Toftenvaag and Tande (1989), which also comprizes a dumpload and a short term battery storage through a line-frequency coverter. The system is operated in different modes according to the actual wind and load power and the state of charge of the battery storage. In this work we focus on the wind-diesel mode.

As seen from Fig. 1, there are two control inputs, the field excitation of the synchronous generator $\left(u_{f}\right)$, and the fuel flow input to the diesel engine $\left(q_{f}\right)$. There is no active control of the wind turbine. The windspeed $\left(v_{w}\right)$ and the load impedance $\left(R_{L}\right)$ can be regarded as plant disturbances. The bus-bar voltage amplitude $(U)$ and the angular speed of the diesel engine $(\omega)$ are chosen as the main measurements of output variables.

\subsection{Wind-diesel modeling}

A nonlinear dynamic model of the system in Fig. 1 is developed using the software tools described in Lundsager et al. (1991), Uhlen et al. (1993) where also validation results are presented. The model is of the form:

$$
\begin{aligned}
& \dot{x}=f(x, z, v)+B u \\
& 0=g(x, v) z+b(x) \\
& y=h(x, z) \\
& u=\left[u_{1}, u_{2}\right]^{\mathrm{T}}=\left[u_{f}, q_{f}\right]^{\mathrm{T}} \\
& y=\left[y_{1}, y_{2}\right]^{\mathrm{T}}=[U, \omega]^{\mathrm{T}}
\end{aligned}
$$

The nine differential equations describe the rate of change of the electric machine rotor fluxes, the main dynamic modes of the mechanical drive trains, and the actuator dynamics. The algebraic equations, $g(\cdot)$, are results of neglecting the fast dynamics of the stator fluxes. This equation may then be viewed as the electrical load flow model, where the unknowns, $z$, are the transformed stator current and voltage components of the two electrical machines. $v$ represents the disturbances (windspeed and load impedance), $u$ the control inputs, and $y$ the measurement vector.

An important feature of the model is the ability to describe magnetic saturation of the electrical machines, which may have considerable impact on the dynamic behaviour of this kind of systems.

\subsection{Linearized models}

Linear plant models must be developed for the design of some model based controllers, like the LQG/LTR-controller, and for linear system analyses. For this purpose, the full nonlinear model (1) is linearized analytically:

$$
\begin{aligned}
& \dot{x}=A x+B u+E v \\
& y=C x
\end{aligned}
$$

where:

$$
\begin{aligned}
A & =\frac{\partial f}{\partial x}+\frac{\partial f}{\partial z} \frac{\Delta z}{\Delta x} \quad E=\frac{\partial f}{\partial v}+\frac{\partial f}{\partial z} \frac{\Delta z}{\Delta v} \\
C & =\frac{\partial h}{\partial x}+\frac{\partial h}{\partial z} \frac{\Delta z}{\Delta x} \\
\frac{\Delta z}{\Delta x} & =-\frac{g^{-1} b\left(x_{0}+\Delta x\right)-g^{-1} b\left(x_{0}\right)}{\Delta x}
\end{aligned}
$$


The partial derivatives are computed as chosen operating points $\left(x_{0}, u_{0}, v_{0}\right)$.

\subsection{Reduced order model}

A reduced order model is used for the design of a feedback linearization controller. This 2 nd order model is derived by neglecting fast dynamic modes associated with drive train flexibilities, combustion dynamics and electrical dynamics of the induction machine. The model is on the desirable companion form:

$$
\begin{aligned}
& \dot{x}=f(x, v)+B u \\
& y=h(x)
\end{aligned}
$$

Robust stability and performance analysis is a well established methodology for investigating dynamic system behaviour, when explicitly taking into account model imperfections and uncertainties. Some degree of model uncertainty will always be present, and in this case we may consider the three main sources of uncertainty as:

- Uncertain or time varying model parameters.

- Nonlinearities, e.g. simplified or non-modeled description of saturation in electrical machine and actuators.

- Non-modeled dynamics, e.g. from neglecting the stator and damper winding dynamics, simplified modeling of drive trains or simplified combustion and aerodynamic models.

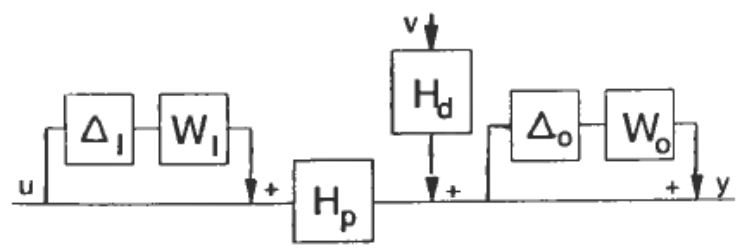

Figure 2. Model uncertainty description. $H_{p}(j \omega)=C(j \omega I-A)^{-1} B$-Nominal plant model. $H_{p}(j \omega)=C(j \omega I-A)^{-1} E-$ Nominal disturbance model.

For linear system analysis, the uncertainties are modeled as input and output uncertainty using the norm-bounded perturbation model, $\Delta(j \omega)$, as illustrated in Fig. 2. Input uncertainty is associated mainly with uncertain actuator gain and dynamics, while all other model imperfections are lumped into the output uncertainty. The perturbation model represents any multivariable transfer function with principal gains less than one at all frequencies, i.e. it satisfies:

$$
\sigma_{i}(\Delta(j \omega))=\left(\lambda_{i}\left(\Delta^{H} \Delta\right)\right)^{1 / 2} \leq 1 \quad \forall \omega \geq 0, i \in\{1,2\}
$$

$\sigma_{i}$ are singular values. The bounds on the model uncertainty are described by the weighting functions, $w_{I}(j \omega)$ and $w_{0}(j \omega)$. The following transfer functions are used based on analysis of the family of linearized plants:

$$
w_{I}(s)=0.1 \frac{1+0.1 s}{1+0.02 s} I_{2} \quad w_{0}(s)=0.3 \frac{1+0.05 s}{1+0.003 s} I_{2}
$$

$I_{2}$ is the 2-by-2 identity matrix. These error models imply that the model error is small at low frequencies, increasing and eventually saturating at higher frequencies. 
Generally this method does not constitute a tight description of the 'real' uncertainty. For this system it may not be realistic to use a coupled description of the input uncertainty, since the control inputs are mainly independent. A more detailed description and discussion of this methodology can be found e.g. in Morari and Zafiriou (1989).

\section{Control objectives and controller design}

The control objectives should be reflected in the system performance specification. Several objectives may be specified in this case:

- Power quality specifications, i.e. minimizing voltage and frequency deviations. This implies a high closed loop system bandwidth.

- Minimization of mechanical torque transients in the drive trains.

- Robustness, i.e. the controller should give adequate performance for all operating points in which it is active.

There is a conflict between high bandwidth requirements, on one hand, and sufficient stability margins on the other hand. This trade-off can be reflected if the system performance specification is described as a frequency dependent function of the control error, $e=y_{\text {ref }}-y$. A performance weight can be constructed such that our control objectives are satisfied if:

$$
\begin{gathered}
\|\epsilon(j \omega)\|=\left\|w_{p}(j \omega) e(j \omega)\right\| \leq 1 \quad \forall \omega \geq 0 \\
w_{p}(s)=200 \frac{1+0.33 s}{1+3 \cdot 3 s} I_{2}
\end{gathered}
$$

The chosen function, $w_{p}(j \omega)$ corresponds to the following performance specifications:

- Maximum $0.5 \%$ frequency and voltage deviations allowed at steady state.

- Desired closed loop system bandwidth of about $3 \mathrm{rad} / \mathrm{s}$.

- Absolute maximum allowed transient frequency and voltage deviations are 5\%.

In order to achieve the specified performance, a variety of controller design methods may be chosen. Three basically different control structures are chosen for this study. The first is an inverse-based controller consisting of a feedback linearization compensator in parallel with diagonal PI-control, (Foss and Johansen (1992)), as illustrated in Fig. 3. The motivation behind this structure is that it provides a simple and robust solution to the use of decoupling control. It is also seen that the structure of the final controller is very similar to a standard turbine and generator controller, that includes some kind of power system stabilizer. This controller depends on the state, $x$. We will use the low order model (4), and in this case the states are measurable. Hence, there is no need for a state estimator or observer.

The LQG/LTR-design is chosen because it provides a simple method for designing a robust multivariable controller with a structure as shown in Fig. 4. With minor modifications, the design procedure follows closely that described in Athans (1986), and represents a combination of frequency domain loop shaping, and result from optimal control theory.

Ordinary single loop controllers are chosen for comparison purposes, and since this is the way most diesel generator sets are controlled. 


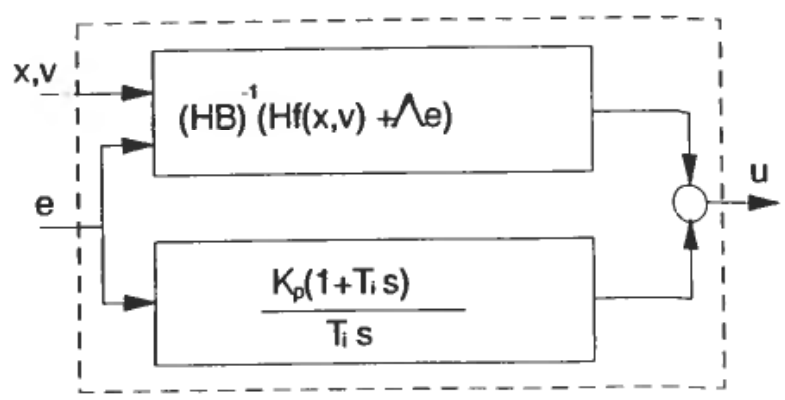

Figure 3. Structure of the 'FLPI-controller'.

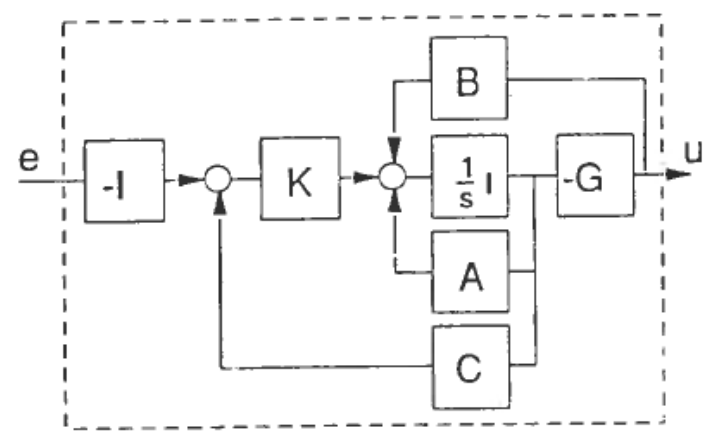

Figure 4. Structure of the LQG/LTR-controller.

\section{System analysis and simulation study}

\subsection{Plant analysis}

The open loop plant model is linearized at seven operating points, where the operating points are defined at nominal voltage and angular speed, and in terms of different windspeed and load conditions, as shown below.

\begin{tabular}{lccc}
\hline & \multicolumn{3}{c}{ Consumer load } \\
\cline { 2 - 4 } Windspeed & $0 \mathrm{~kW}$ & $25 \mathrm{~kW}$ & $50 \mathrm{~kW}$ \\
\hline $4 \mathrm{~m} / \mathrm{s}$ & 1 & 2 & 3 \\
$7 \mathrm{~m} / \mathrm{s}$ & & 4 & 5 \\
$10 \mathrm{~m} / \mathrm{s}$ & & & 6 \\
$13 \mathrm{~m} / \mathrm{s}$ & & & 7 \\
\hline
\end{tabular}

Operating point 4 is chosen as the nominal plant model. The two singular value plots of the open loop frequency response matrices from $\mathrm{u}$ to $\mathbf{y}, \sigma_{i}\left(H_{p}(j \omega)\right)$, for all seven plants are shown in Fig. 5. Large gain variations are observed in the medium and low frequency range, which clearly indicate the nonlinear nature of the system. Note also the resonant peak at about $20 \mathrm{rad} / \mathrm{s}$, which may cause problems if too high bandwidth is imposed on the closed loop system.

To gain some insight in the multivariable aspects of the plant, one possible approach is to study the relative gain array (Bristol (1966)). The relative gains, $\lambda_{\mathrm{ij}}$ of a multivariable system $y_{i}=\{h\}_{i j} u_{j}$ are defined as the ratio between the open loop gain 


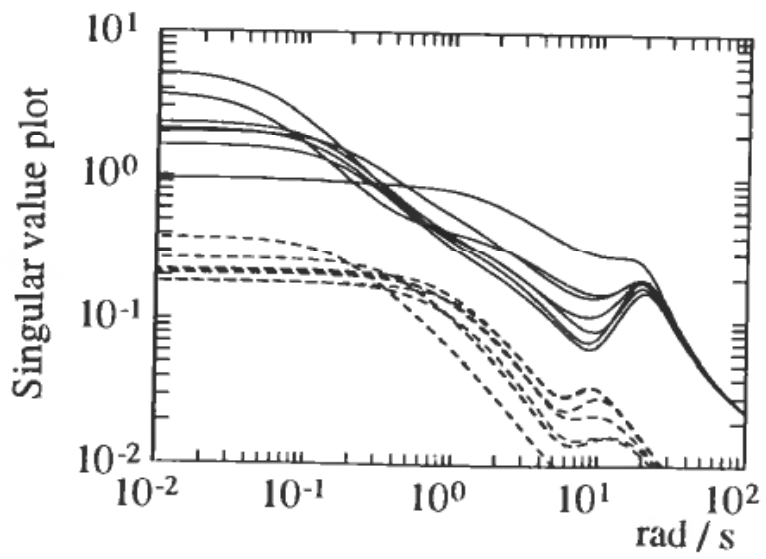

Figure 5. Singular value plots of the 7 linearized plants.

from $u_{j}$ to $y_{i}, h_{i j}$, and the corresponding gain when all outputs, except $y_{i}$, are perfectly controlled. The relative gain array (the matrix of all the relative gains) is computed as

$$
\Lambda_{r g a}(j \omega)=H_{p} \times\left[H_{p}^{-1}\right]^{\mathrm{T}}
$$

where $\times$ denotes element by element multiplication, For a $2 \times 2$ system, the relative gain array is simply:

$$
\Lambda_{r g a}(j \omega)=\left[\begin{array}{cc}
\lambda_{11} & 1-\lambda_{11} \\
1-\lambda_{11} & \lambda_{11}
\end{array}\right] \lambda_{11}(j \omega)=\frac{1}{1-\frac{h_{12} h_{21}}{h_{11} h_{22}}(j \omega)}
$$

The relative gains contain information about the couplings between inputs and outputs of the system, and are useful as an aid to choose pairing of single loop controllers. A perfectly decoupled system will have $\lambda_{11} \in\{0,1\}$. Very large relative gains represent an ill-conditioned system, which makes the use of inverse-based controllers impossible because of high sensitivity with respect to control input errors (Skogestad and Hovd (1990)).

The real parts of the frequency dependent relative gains for the plant models at three different windspeeds are shown in Fig. 6. Information gained from these plots can be summarized as follows:

- The couplings in $H_{p}$ are small, around and above the bandwidth frequency $(\approx 3 \mathrm{rad} / \mathrm{s})$. Hence, the need for a decoupling controller is limited in this frequency range.

- There are stronger couplings in the intermediate and low frequency range, and the couplings tend to be stronger at higher windspeeds. This indicates that there may be something to gain by decoupling. A main question is how effective the decoupler can be made using the reduced order model.

- The relative gains are not seen to be very large for any of the linearized plants. No serious robustness problems are therefore expected by inverse-based control, such as the FLPI-controller.

- The normal pairing for single loop controllers is correct, $\lambda_{11}(j \omega)$ close to one in most cases. There might, however, be problems at high windspeeds. 


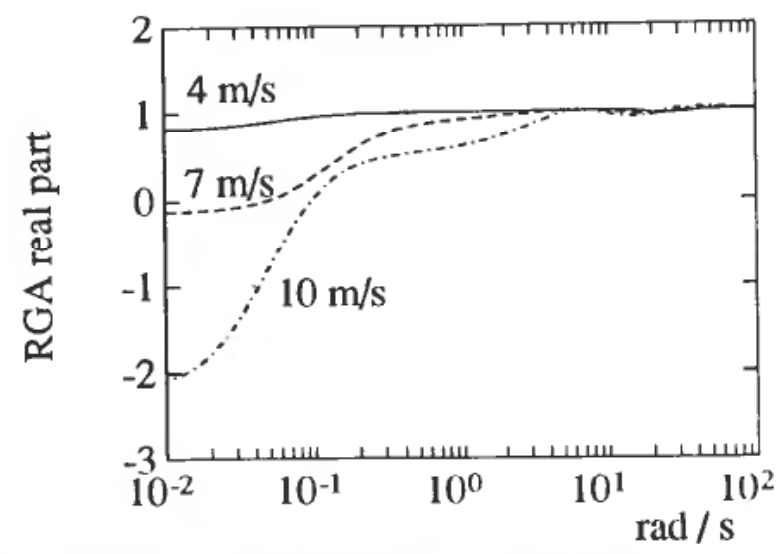

Figure 6. Relative gains of linearized plants at three operating points.

\subsection{Controller design}

The feedback linearization part of the FLPI-controller is derived directly from the reduced model (4). The aim of this compensator is to obtain a linear and decoupled closed loop system:

$$
\dot{y}=\Lambda\left(y_{r e f}-y\right) \quad \Lambda=\operatorname{diag}\left(\lambda_{1}, \lambda_{2}\right)
$$

This is obtained by the followed compensator, where $\Lambda$ are design parameters for specifying desired bandwidth:

$$
u_{F L}=\left(\frac{\partial h}{\partial x} B\right)^{-1}\left[\frac{\partial h}{\partial x} f(x, v)+\Lambda\left(y_{r e f}-y\right)\right]
$$

By inserting the model equations (4) and linearizing the nonlinear terms, the final compensator was chosen as:

$$
u_{F L}=\left[\begin{array}{l}
u_{f} \\
q_{f}
\end{array}\right]=\left[\begin{array}{lr}
4.3 & -5.0 \\
0 & 1.9
\end{array}\right]\left[\begin{array}{l}
U_{r e f}-U \\
\omega_{r e f}-\omega
\end{array}\right]+\left[\begin{array}{cl}
-2.3 & 0 \\
0 & 0.4
\end{array}\right]\left[\begin{array}{l}
i_{s d} \\
P_{s g}
\end{array}\right]
$$

The PI-controller, as the second part of the FLPI-controller, were chosen as:

$$
u_{P I}=\left[\begin{array}{cc}
11.7 & 0 \\
0 & 10
\end{array}\right]\left(e+\left[\begin{array}{ll}
1.5 & 0 \\
0 & 1.5
\end{array}\right] \int_{0}^{t} e d t\right)
$$

The controller is seen to depend on two additional variables, the electrical power and the d-axis current components of the synchronous machine. These variables can easily be measured or estimated, and do not represent a problem for application of this control structure. On the other hand, the contribution from these measurements can be viewed as a feed-forward term, and thereby improve system performance. The first part of the controller (12) is essentially a detuned decoupler. At low frequencies, the controller is almost diagonal, since the integral terms of the PI-controllers dominate.

Design of the LQG/LTR-controller follows the dual approach to the procedure described in Zhang and Freudenberg (1990). Integrators are augmented at the plant input, and the target feedback loop

$$
H_{t f}=\tilde{C}(s I-\tilde{A})^{-1} K
$$


is obtained from the following design specifications $(\bar{A}, \bar{B}, \bar{C}$ represent the augmented plant model):

- Equal, high gains at low frequencies to improve wind disturbance attenuation, $\sigma_{i}\left(H_{t f}\right) \approx 45 / \omega i \in\{1,2\}$.

- Equal, but relatively lower gains at high frequencies, to avoid excitation of the main eigenfrequency, $\sigma_{i}\left(H_{t f}\right) \approx 10 / \omega$. The desired system bandwidth is then $\approx 10 \mathrm{rad} / \mathrm{s}$.

The final controller is obtained from the loop transfer recovery, i.e. a controller gain matrix, $G(\rho)$ is found such that:

$$
\lim _{\rho \rightarrow 0}\left[H_{p} H_{c}(\rho)\right] \rightarrow H_{t f}
$$

where $H_{p}$ represent the plant model, and $H_{c}$ is the controller (Fig. 4). $G(\rho)$ is found from the algebraic Riccati equation:

$$
\begin{aligned}
X \tilde{A}+\tilde{A}^{\mathrm{T}} X-\frac{1}{\rho} X \tilde{B} \tilde{B}^{\mathrm{T}} X+\tilde{C}^{\mathrm{T}} \tilde{C} & =0 \\
G & =\frac{1}{\rho} \tilde{B}^{\mathrm{T}} X
\end{aligned}
$$

The final controller is reduced to an 8th order compensator by applying standard model reduction techniques, which is an acceptable order, also for practical implementations.

All these controllers, including the single loop PI-controllers, are tuned similarly with respect to desired system bandwidth.

\subsection{Linear frequency domain system analysis}

The resulting nominal open loop frequency responses, using the three different controllers, are shown Fig. 7. The medium and crossover frequency range from 1 to $10 \mathrm{rad} / \mathrm{s}$ is found to be the most critical with respect to system performance. The following observations are important:

For the FLPI-controller, the narrow band between the upper and lower singular values in the medium frequency range indicates that decoupling, to some degree, is effective in this frequency range. The lower singular value is lifted, compared to the PI-controller case, and thus the closed loop bandwidth should be increased. Poorer robustness is not expected since the upper singular values are not very different in the two cases.

A somewhat different response is observed for the LQG/LTR-controller. The larger band between upper and lower singular values shows that decoupling is not present to any large degree. The lower singular value indicates a bandwidth equal to the FLPI-controller case. The upper singular value is seen to be very large, which may affect the robustness of the closed loop system. Equal singular values, as specified in the design, are obtained only in the very low frequency range.

Figure 8 illustrates how the transfer function block model of the closed loop system is transformed to the $\mathbf{M}-\Delta$ description, which is used for numerical frequency domain analyses (See Appendix 1). The closed loop system performance is defined as the system's ability, in terms of voltage and angular speed variations, to reject disturbances from windspeed and load variations. Mathematically, this is analysed by studying the 


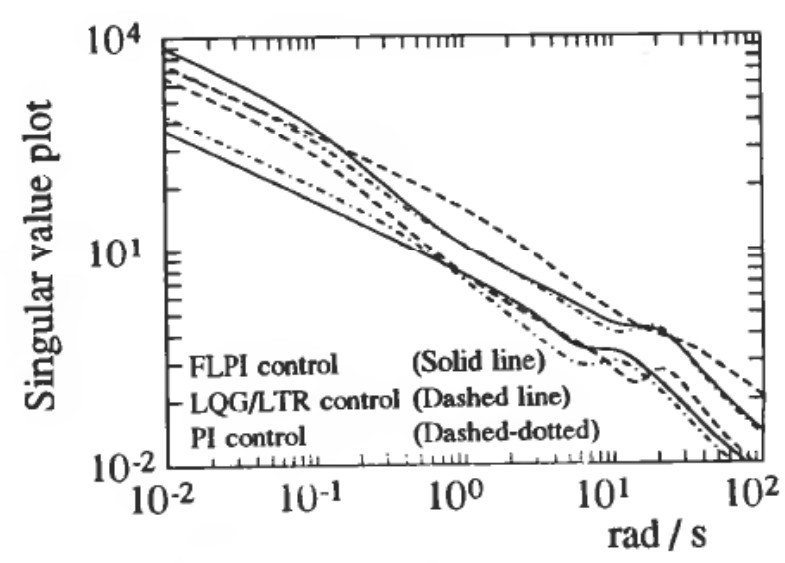

Figure 7. Open loop singular value plots.

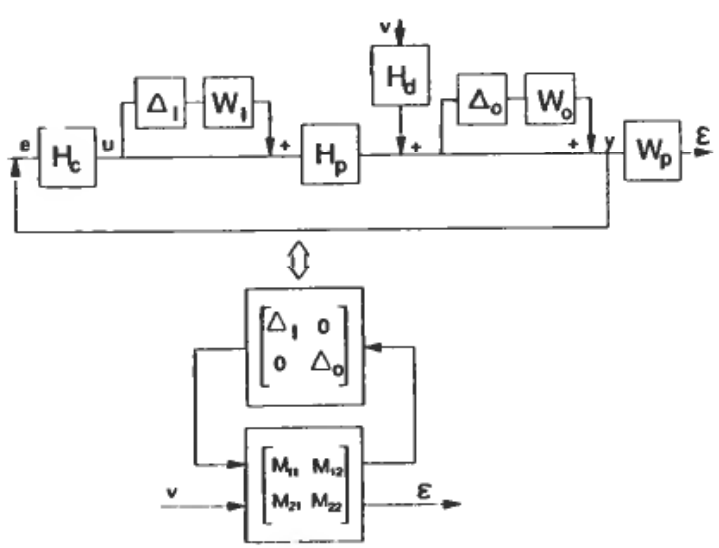

Figure 8. Closed loop system.

transfer function matrix for $v$ to $\varepsilon(7)$. When neglecting model uncertainty, the transfer function is:

$$
\epsilon(j \omega)=w_{p}\left(I+H_{p} H_{c}\right)^{-1} H_{d} v(j \omega)=M_{22} v(j \omega)
$$

$H_{c}(j \omega)$ represents the actual controller. The maximum singular value of $M_{22}(j \omega)$, using the three controllers are shown in Fig. 9. This represents the nominal performance of the system. It is observed that:

- All controllers yield satisfactory performance since the singular values do not exceed one.

- The FLPI-controller provides better disturbance rejection than the other controllers in the whole frequency range.

- The LQG/LTR-controller is nominally better than the PI-controller in the medium and higher frequency range.

The purpose of the robust stability analysis, is to investigate whether the nominally stable closed loop system is stable, also in the presence of model uncertainty as defined in Fig. 2 and (6). A system is said to be robust stable if it is stable for all the possible plants resulting from the model uncertainty description. The analysis involves 


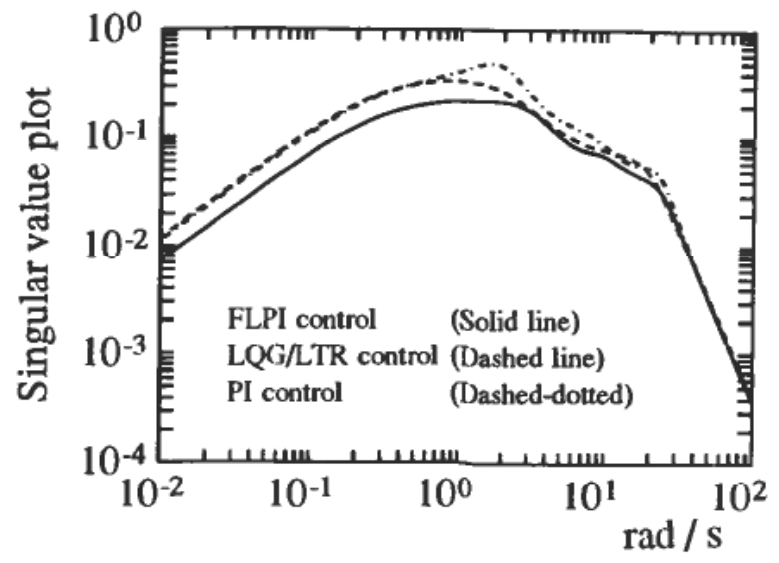

Figure 9. Nominal performance analysis showing $\sigma_{\max }\left(M_{22}\right)$.

computation of the structured singular value, $\mu$, (Morari and Zafirou (1989)), and the stability criterion is, assuming that the nominal system is stable:

$$
\mu\left(M_{11}(j \omega), \Delta(j \omega)\right)<1 \quad \forall \omega \geq 0
$$

$M_{11}(j \omega)$ is the resulting transfer function matrix when rearranging the block diagram as illustrated in Fig. 8 (and Appendix 1). The structured singular values, computed for the wind-diesel system with the three different controllers, are shown in Fig. 10.

This analysis shows that the PI- and FLPI-controllers yield similar stability properties, and both systems are seen to be robust stable. For the LQG/LTR-controller case, the system is not robust stable at all frequencies. This problem was also indicated by the open loop frequency responses (Fig. 7), and illustrates that this controller is more sensitive to coupled (directional) uncertainty. However, this analysis represents the worst case, and the uncertainty description may give rise to unphysical plants. The input uncertainty is not likely to be coupled, as assumed in this analysis. This may very well change the conclusion with respect to robust stability.

A measure of the robust performance is found by computing the structured singular value of the full rearranged system matrix, $M(j \omega)$. Our performance criterion is:

$$
\mu(M(j \omega), \Delta(j \omega))<1 \quad \forall \omega \geq 0
$$

This measure illustrates how the nominal system performance deteriorates because of model uncertainty. The robust performance plots of Fig. 11 are seen to confirm the information gained from the nominal performance and robust stability analyses:

- Relatively less good performance, compared to the nominal case, for all controllers, due to model uncertainty.

- The difference in performance between the controllers seem to have become smaller in the low and medium frequency range, but still the FLPI-controller is the best choice.

- In the high frequency range, the poorer stability properties of the LQG/LTRcontroller is also reflected in the robust performance.

- The high frequency stability problem of the LQG/LTR-controller may be explained as a result of a too conservative input uncertainty description. If this is the case, we can conclude that both the suggested controllers provide improved performance compared to single loop PI-controllers. 


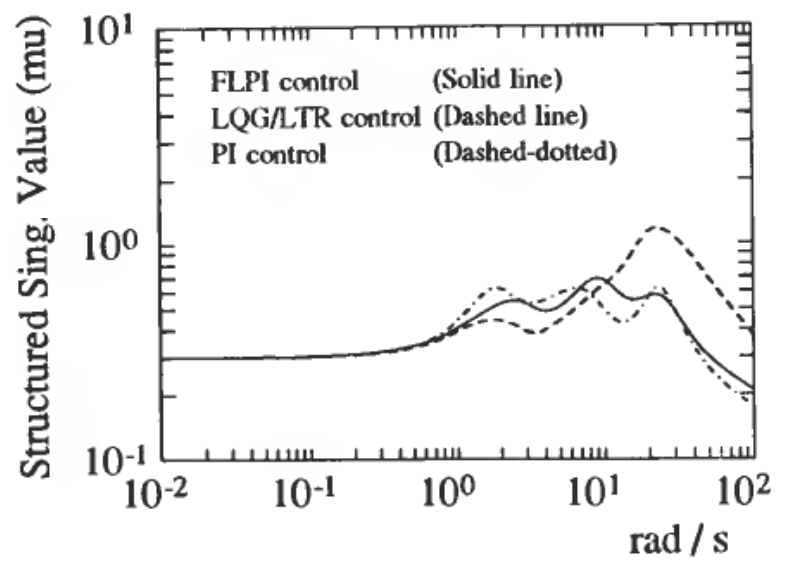

Figure 10. Robust stability analysis showing $\mu\left(M_{11}\right)$.

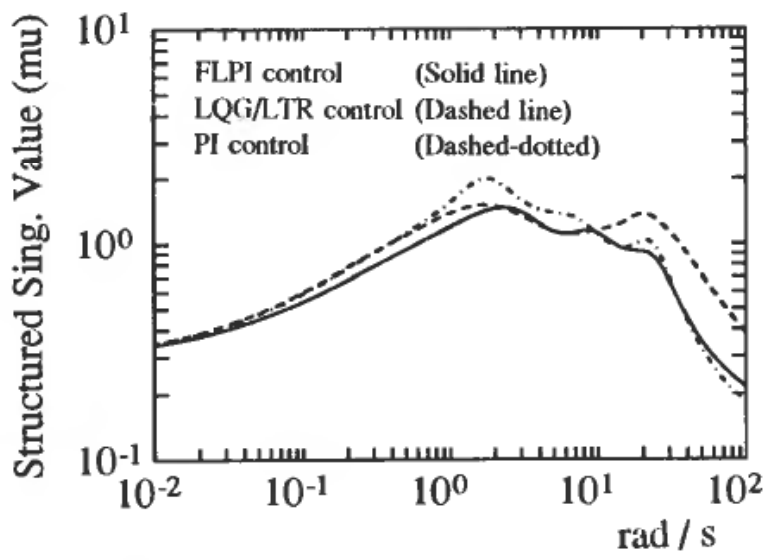

Figure 11. Robust performance analysis showing $\mu(M)$.

\subsection{Simulation study}

A main objective of this study has been to assess linear multivariable analysis techniques, and how reliable results can be expected when such techniques are applied to a nonlinear power system. For this purpose, a comprehension simulation study has been carried out for comparison of the results. The full nonlinear simulation model is used to analyse the system's performance at different operating points in the time domain. Since this model has been validated with measurements from the actual system, there is little uncertainty associated with this simulator.

A few representative results are presented here to show that the simulations agree well with the results from the linear analysis. Figures 12 and 13 show the system's response to a step change in load impedance corresponding to a step in consumer load power form 25 to $35 \mathrm{~kW}$ (Fig. 12). The windspeed is constant $7 \mathrm{~m} / \mathrm{s}$, and thus this case represents a disturbance of the 'nominal' system. 


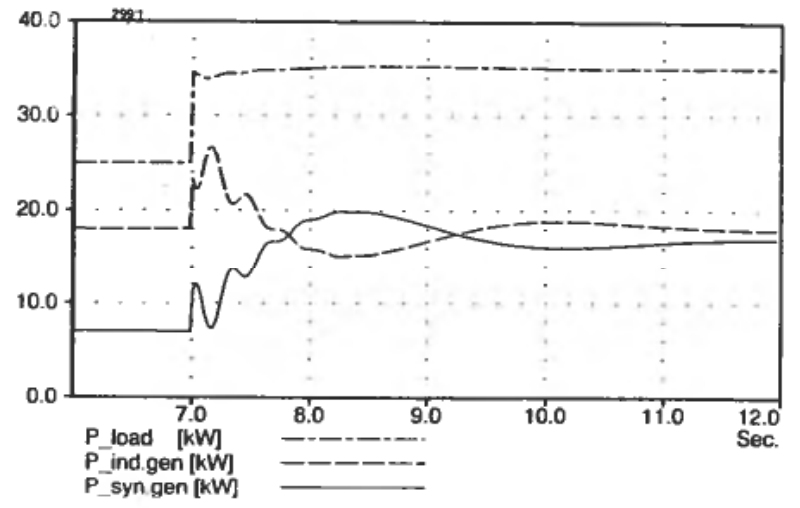

Figure 12. Electrical power distribution.

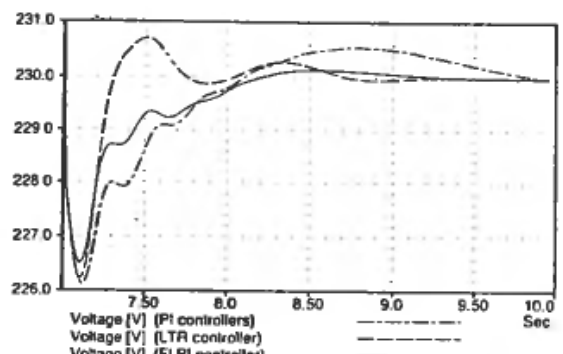

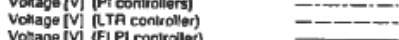

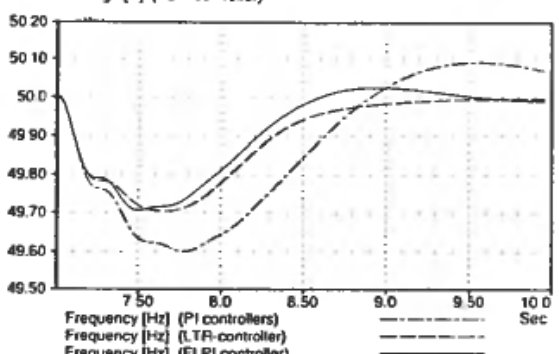

Figure 13. Bus-bar voltage and frequency responses.
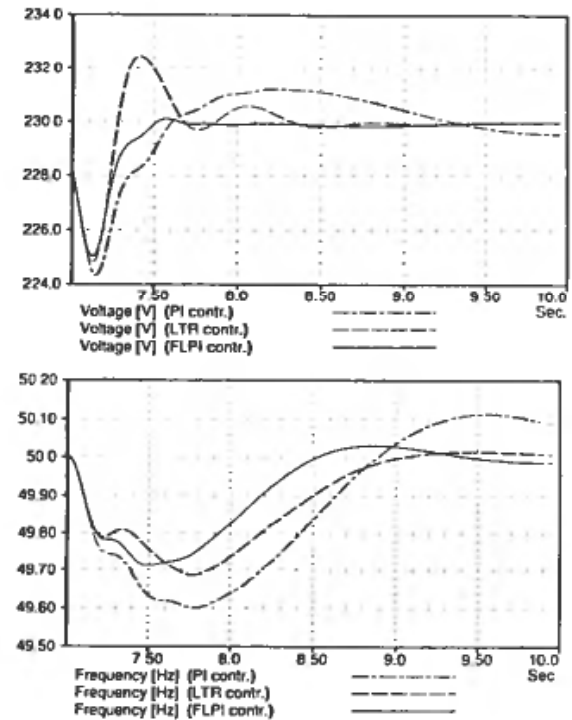

Figure 14. Bus-bar voltage and frequency responses. 
Figure 14 shows responses to a similar step in load from 35 to $45 \mathrm{~kW}$ at $10 \mathrm{~m} / \mathrm{s}$ windspeed. The first plots (Figs. 12 and 13) show that the best performances are provided by the FLPI-controller and the LQG-LTR-controller. Comparisons between the two operating points (Figs. 13 and 14) show that the responses with the FLPI-controller are very similar in the two cases, while the performance of the LQG/LTR-controller has declined in the latter case. This is seen as a slower angular speed response and a larger overshoot in grid voltage. This results are thus in good agreement with the conclusion from the robust performance analysis.

\section{Conclusion}

Multivariable frequency domain techniques have been applied to controller design and analysis of a wind-diesel power plant.

It is shown how three different robust control structures yield different results with respect to stability and performance of the nonlinear and multivariable system. Conclusions from the frequency domain analyses are in good agreement with results obtained from detailed nonlinear simulation studies.

It is therefore hoped that this work can contribute to introduce multivariable frequency domain techniques as an interesting and applicable option for dynamic power system analyses.

With respect to performance of the suggested controllers, this work shows that noticeable improvements, compared to single loop PI-controllers, can be achieved by the composite feedback linearization/PI-controller structure. Improved performance may also be achieved by the LQG/LTR method, but some work is needed to develop a sufficiently simple and robust implementation of this controller.

\section{ACKNOWLEDGMENTS}

The authors thank the Norwegian Water Resources and Energy Administration (NVE), the Research Council of Norway (NFR-NTNF) and EFI for supporting this work.

\section{Appendix 1}

The transformed closed loop frequency response matrix used in the computation of robust stability and performance is given by:

$$
M(j \omega)=\left[\begin{array}{cc|c}
-\left(I+H_{c} H_{p}\right)^{-1} H_{c} H_{p} w_{I}-\left(I+H_{c} H_{\mathrm{p}}\right)^{-1} H_{c} w_{0} & -\left(I+H_{c} H_{p}\right)^{-1} H_{c} H_{d} \\
\left(I+H_{p} H_{c}\right)^{-1} H_{p} w_{I}-\left(I+H_{p} H_{c}\right)^{-1} H_{c} w_{0} & \left(I+H_{p} H_{c}\right)^{-1} H_{d} \\
\hdashline-\frac{1}{w_{p}\left(I+H_{p} H_{c}\right)^{-1} H_{p} w_{I}}-\frac{-1}{w_{p}\left(I+H_{p} H_{c}\right)^{-1}}-w_{0} & w_{p}\left(I+H_{p} H_{c}\right)^{-1} H_{d}
\end{array}\right]
$$

\section{REFERENCES}

Athans, M. (1986). A tutorial on the LQG/LTR method. In American Control Conference, Seattle, WA, June, 1986.

BRISTOL, E. H. (1966). On a new measure of interaction for multivariable process control. IEEE Trans. Automatic Control, 11, 133-134.

Foss, B. A., and JOHANSEN, T. A. (1992). Parallel nonlinear decoupling for process control-a NARMAX approach, IFAC/IFIP/IMACS International Symposium-Artificial Intelligence in Real-Time Control, 1992 (invited paper). pp. 209-214.

INFIELD, D. G. et al. (1992). Wind diesel systems - design assessment and future potential. Wind Engineering, 16. 
LundSAGer, P. et al. (1991). Progress with the European wind-diesel modeling software package. In Proc. EWEC'91 (European Wind Energy Conference), Amsterdam, 1991. MORARI and ZAFIRIOU (1989). Robust Process Control (Prentice-Hall International Editions), pp. 248-252.

SKogestad, S. and Hovd, M. (1990). Use of frequency-dependent RGA for Control Structure Selection. Proc. American Control Conference, ACC'90, San Diego, 1990.

Uhlen, K., Skarstein, Ø., ToftevaAG, T., and TANDE, J. O. G. (1989). Design and operation of the full scale Norwegian wind/diesel laboratory model. Proc. EWEC'89, Glasgow, 1989.

UHLEN, K. et al. (1993). Engineering design tools for wind-diesel systems: presentation and validation of the modular dynamic model. Proc. ECWEC'93 (European Community Wind Energy Conference), Travemunde, Germany, 1993.

ZHANG and FREUDENBERG (1990). Loop transfer recovery for nonminimum phase plants. IEEE Trans Automatic Control, 35. 